\title{
Integral quadratic forms and Dirichlet series
}

\author{
B. van Asch • F. van der Blij
}

Received: 22 October 2008 / Accepted: 22 December 2009 / Published online: 19 March 2010

(C) The Author(s) 2010. This article is published with open access at Springerlink.com

\begin{abstract}
A Dirichlet series with multiplicative coefficients has an Euler product representation. In this paper we consider the special case where these coefficients are derived from the numbers of representations of an integer by an integral quadratic form. At first we suppose this quadratic form to be positive definite. In general the representation numbers are not multiplicative. Instead we consider the average number of representations over all classes in the genus of the quadratic form. And we consider only representations of integers of the form $t k^{2}$ with $t$ square-free. If we divide the average representation number for these integers by a suitable factor, we do get a multiplicative function. Using results from Siegel (Ann. Math. 36:527-606, 1935), we derive a uniform expression for the Euler product expansion of the corresponding Dirichlet series. As a special case, we consider the standard quadratic form in $n$ variables corresponding to the identity matrix. Here we use results from Shimura (Am. J. Math. 124:1059-1081, 2002). For $2 \leq n \leq 8$, the genus of this particular quadratic form contains only one class, and this leads to a rather simple expression for the Dirichlet series, where the coefficients are just the number of representations of a square as the sum of $n$ squares. Finally we consider the indefinite case, where we can get results similar to the definite case.
\end{abstract}

Keywords Integral quadratic form · Multiplicative function - Dirichlet series · Euler product

Mathematics Subject Classification (2000) Primary 11B34 · 11E25 · 11F66 • $11 \mathrm{~K} 65$

B. van Asch $(\bowtie)$. F. van der Blij

Department of Mathematics and Computing Science, Eindhoven University of Technology,

P.O. Box 513, 5600 MB Eindhoven, The Netherlands

e-mail: a.g.v.asch@tue.nl 


\section{Introduction}

A classical problem in number theory is the determination of the number of representations of a positive integer $k$ as a sum of $n$ squares of integers. In the case $n=2$ this number $r_{2}(k)$ can be calculated using complex numbers, more special Gaussian integers. In the cases $n=4,6$, and 8 , this number can be found using complex analysis such as the theory of modular forms. The coefficients of powers of the theta functions are equal to the number of representations as a sum of squares. The resulting formulae for $r_{n}(k), n=2,4,6,8$, are classic and can, for instance, be found in [1] and [2]. The number-theoretic functions $r_{n}(k)$ are not multiplicative, but for $n=2,4,8$, the functions $\rho_{n}(k)=\frac{1}{2 n} r_{n}(k)$ are. However, the function $\rho_{6}(k)$ is not multiplicative. From [2] we can get formulas for $r_{3}(k), r_{5}(k)$, and $r_{7}(k)$, too. It appears that if for $n=2, \ldots, 8$, we consider $\rho_{n}\left(k^{2}\right)$ instead of $\rho_{n}(k)$, we get a multiplicative function. And when we use these functions to define Dirichlet series, these series can be expressed as an infinite product. But this stops at $n=8$. In this paper we will generalize these results to the case $n>8$. We have to modify the definition of the function $\rho_{n}(k)$. First we make some general remarks about Dirichlet series and Euler products. Then we consider positive definite integral quadratic forms, and we will use results from Siegel [4]. Thereafter we specialize this quadratic form to the original question, i.e., the sum of $n$ squares. For this part, we will use results from [2]. And finally, using results from Siegel again (see [5] and [6]), we will consider indefinite quadratic forms and get results similar to the definite case.

\section{Dirichlet series and Euler products}

In its simplest form a Dirichlet series is a series of the form $D(s)=\sum_{n=1}^{\infty} \frac{a_{n}}{n^{s}}$. If $a_{n}=O\left(n^{c}\right)$, then $D(s)$ converges absolutely and uniformly for $\operatorname{Re}(s) \geq c+1+\varepsilon$. It is well known that if the coefficients $a_{n}$ are multiplicative, i.e., $a_{m n}=a_{m} a_{n}$ whenever $\operatorname{gcd}(m, n)=1$, then $D(s)$ has an Euler product representation,

$$
D(s)=\prod_{p}\left(\sum_{j=0}^{\infty} \frac{a_{p^{j}}}{p^{s j}}\right) .
$$

In particular we can take for the $a_{n}$ the Fourier coefficients of some modular form $f(z)$. In that case the corresponding Dirichlet series has an Euler product representation if and only if the modular form is a common eigenfunction for certain Hecke operators. In his work on modular forms of half integral weight, Shimura gave an Euler product for the Dirichlet series $D(s)=\sum_{n=1}^{\infty} \frac{a\left(t n^{2}\right)}{n^{s}}$, where $t$ is a positive square-free integer, and $f(z)=\sum_{n=0}^{\infty} a(n) e^{2 \pi i n z}$ is a modular form for a subgroup of the modular group, which is a common eigenfunction for some specific Hecke operators (see [3], Theorem 1.9). It can be proved that the average theta series corresponding to the coefficients we will consider in Section 4 is such a common eigenfunction. Therefore Shimura's Theorem 1.9 applies, which would have been another way to prove Theorem 3. In a more general setting Siegel modular forms on the Siegel upper halfplane of degree $n$ were considered by Andrianov and Zhuravlev. In particular, they 
considered the weighted average over the genus of the theta series of the forms in the genus. These modular forms are eigenfunctions of all "good" Hecke operators in the even Hecke algebra. For details, see, for instance, [7]. In [8] Zhuravlev presented an explicit Euler product decomposition of the Dirichlet series associated to these particular modular forms. However, in this paper we will not use results from the theory of modular forms. Instead, we start with results from Siegel on global and local representation numbers by quadratic forms. It appears that rather elementary calculations lead to Euler product decompositions of certain Dirichlet series. And with a slight modification this method can even be applied to the case of an indefinite quadratic form.

\section{Positive definite quadratic forms}

Consider the positive definite quadratic form $Q(X)=X^{T} S X$, where $S$ is a positive definite symmetric integral $n \times n$ matrix, and $X=\left(\begin{array}{llll}x_{1} & x_{2} & \ldots & x_{n}\end{array}\right)^{T}$. Denote by $A_{0}(S, t)$ the average number of integral solutions of $Q(X)=t$ over all classes in the genus of $S$. Siegel [4] proved that

$$
A_{0}(S, t)=\alpha_{\infty}(S, t) \prod_{p} \alpha_{p}(S, t)
$$

where $\alpha_{\infty}(S, t)$ is a measure on the real solution space of $X^{T} S X=t$, and for every prime number $p$, the number $\alpha_{p}(S, t)$ represents the local density of the representations of $t$. For all primes $p$ such that $\operatorname{gcd}(p, 2|S|)=1$, there is a very simple expression for these numbers $\alpha_{p}(S, t)$. Write $t=p^{l} t_{1}$ and $\epsilon=\left(\frac{(-1)^{\frac{n}{2}}|S|}{p}\right), r=p^{1-\frac{n}{2}}$ if $n$ is even and $\epsilon=\left(\frac{(-1)^{\frac{n-1}{2}}|S| t_{1}}{p}\right), r=p^{2-n}$ if $n$ is odd. Then we have (see [4], Hilfssatz 16)

$$
\alpha_{p}(S, t)= \begin{cases}\left(1-\epsilon p^{-\frac{n}{2}}\right) \sum_{j=0}^{l} \epsilon^{j} r^{j} & \text { if } n \text { even, } \\ \left(1-p^{1-n}\right) \sum_{j=0}^{\frac{l-1}{2}} r^{j} & \text { if } n \text { odd, } l \text { odd }, \\ \left(1-p^{1-n}\right)\left(\sum_{j=0}^{\frac{l}{2}-1} r^{j}+\frac{r^{\frac{l}{2}}}{1-\epsilon p^{\frac{1-n}{2}}}\right) & \text { if } n \text { odd, } l \text { even. }\end{cases}
$$

Now we consider in particular the numbers $A_{0}\left(S, t k^{2}\right)$, where $t$ is square-free, and $\operatorname{gcd}(2|S|, t)=1$. From [4], Hilfssatz 26 it follows that $\alpha_{\infty}\left(S, t k^{2}\right)=k^{n-2} \alpha_{\infty}(S, t)$. Suppose now that $p$ is a prime such that $p \nmid k$. There is a one-to-one correspondence between the solutions of $X^{T} S X \equiv t k^{2} \bmod p^{a}$ and the solutions of $Y^{T} S Y \equiv$ $t \bmod p^{a}$, and therefore $\alpha_{p}\left(S, t k^{2}\right)=\alpha_{p}(S, t)$. From this we get

$$
\frac{A_{0}\left(S, t k^{2}\right)}{A_{0}(S, t)}=k^{n-2} \prod_{p \mid k} \frac{\alpha_{p}\left(S, t k^{2}\right)}{\alpha_{p}(S, t)} .
$$

Define the function $\rho(S, t, k)$ as $\rho(S, t, k)=\frac{A_{0}\left(S, t k^{2}\right)}{A_{0}(S, t)}$. 
Proposition 1 The function $\rho(S, t, k)$ is a multiplicative function of $k$, i.e., $\rho\left(S, t, k k^{\prime}\right)=\rho(S, t, k) \rho\left(S, t, k^{\prime}\right)$ whenever $\operatorname{gcd}\left(k, k^{\prime}\right)=1$.

Proof This follows in a straightforward way from (2).

As a consequence, the Dirichlet series formed with the coefficients $\rho(S, t, k)$ has an Euler product representation.

\section{Corollary 1}

$$
\sum_{k=1}^{\infty} \frac{\rho(S, t, k)}{k^{s}}=\prod_{p}\left(\sum_{j=0}^{\infty} \frac{\rho\left(S, t, p^{j}\right)}{p^{j s}}\right)
$$

From (2) we get in particular that for $k=p^{j}, \rho\left(S, t, p^{j}\right)=p^{(n-2) j} \frac{\alpha_{p}\left(S, t p^{2 j}\right)}{\alpha_{p}(S, t)}$.

Suppose first that $n$ is even.

If $\operatorname{gcd}(p, 2|S| t)=1$, we get $\rho\left(S, t, p^{j}\right)=\frac{\epsilon p^{1-\frac{1}{2} n}-p^{j(n-2)}}{\epsilon p^{1-\frac{1}{2} n}-1}$, and therefore

$$
\sum_{j=0}^{\infty} \frac{\rho\left(S, t, p^{j}\right)}{p^{j s}}=\frac{1+\frac{\epsilon p^{\frac{1}{2} n-1}}{p^{s}}}{\left(1-\frac{1}{p^{s}}\right)\left(1-\frac{1}{p^{s+2-n}}\right)}
$$

If $p \mid t$, then we get $\rho\left(S, t, p^{j}\right)=\frac{p^{2-n}-p^{j(n-2)}}{p^{2-n}-1}$ and

$$
\sum_{j=0}^{\infty} \frac{\rho\left(S, t, p^{j}\right)}{p^{j s}}=\frac{1}{\left(1-\frac{1}{p^{s}}\right)\left(1-\frac{1}{p^{s+2-n}}\right)}
$$

For the primes $p|2| S \mid$, we put $F(p, 2|S|)=\left(1-\frac{1}{p^{s}}\right)\left(1-\frac{1}{p^{s+2-n}}\right) \sum_{j=0}^{\infty} \frac{\rho\left(S, t, p^{j}\right)}{p^{j s}}$. Combining these expressions, we find that for even $n$, we have

$$
\begin{aligned}
\sum_{k=1}^{\infty} \frac{\rho(S, t, k)}{k^{s}}= & \prod_{p|2| S \mid} F(p, 2|S|) \\
& \times \prod_{p \nmid 2|S| t}\left(1+\frac{\left(\frac{(-1)^{\frac{n}{2}|S|}}{p}\right) p^{\frac{1}{2} n-1}}{p^{s}}\right) \zeta(s) \zeta(s+2-n) .
\end{aligned}
$$

If $n$ is odd, we proceed in the same way. For all $p$ such that $\operatorname{gcd}(p, 2|S| t)=1$, we get

$$
\sum_{j=0}^{\infty} \frac{\rho\left(S, t, p^{j}\right)}{p^{j s}}=\frac{1-\frac{\epsilon p^{\frac{n-3}{2}}}{p^{s}}}{\left(1-\frac{1}{p^{s}}\right)\left(1-\frac{1}{p^{s+2-n}}\right)},
$$


where in this case $\epsilon=\left(\frac{(-1)^{\frac{n-1}{2}}|S| t}{p}\right)$. If $p \mid t$, then we find that

$$
\sum_{j=0}^{\infty} \frac{\rho\left(S, t, p^{j}\right)}{p^{j s}}=\frac{1}{\left(1-\frac{1}{p^{s}}\right)\left(1-\frac{1}{p^{s+2-n}}\right)} .
$$

This leads to the following expression in the case that $n$ is odd:

$$
\begin{aligned}
\sum_{k=1}^{\infty} \frac{\rho(S, t, k))}{k^{s}=} & \prod_{p|2| S \mid} F(p, 2|S|) \\
& \times \prod_{p \nmid 2|S| t}\left(1-\frac{\left(\frac{(-1)^{\frac{n-1}{2}}|S| t}{p}\right) p^{\frac{n-3}{2}}}{p^{s}}\right) \zeta(s) \zeta(s+2-n) .
\end{aligned}
$$

Since $\left(\frac{t^{n}}{p}\right)=1$ for even $n$ and $\left(\frac{t^{n}}{p}\right)=\left(\frac{t}{p}\right)$ for odd $n$, we can combine (3) and (4) to get the following theorem.

\section{Theorem 2}

$$
\begin{aligned}
\sum_{k=1}^{\infty} \frac{\rho(S, t, k)}{k^{s}}= & \prod_{p|2| S \mid} F(p, 2|S|) \\
& \times \prod_{p \nmid 2|S| t}\left(1+(-1)^{n} \frac{\left(\frac{(-1)^{\left\lfloor\frac{n}{2}\right\rfloor}|S| t^{n}}{p}\right) p^{\left\lfloor\frac{n}{2}\right\rfloor}}{p^{s+1}}\right) \zeta(s) \zeta(s+2-n) .
\end{aligned}
$$

\section{Sums of squares}

In this section we take $S=I$, the identity matrix. In [2] Shimura gave explicit formulas for $A_{0}(I, h)$. In the case that $n=2 m+1>3$ is an odd integer, this formula reads as follows:

$$
\begin{aligned}
A_{0}(I, h)= & \frac{2^{n} m(m-1) !(2 \pi)^{-m} L\left(m, \psi_{h}\right)\left(2^{m}-\psi_{h}(2)\right)}{\left|B_{n-1}\right|\left(2^{2 m}-1\right)} \cdot g^{\frac{n-2}{2}} \cdot \tilde{b}(h) \\
& \times \prod_{p \mid r}\left\{\sum_{i=0}^{\lambda_{p}} p^{i(2-n)}-\psi_{h}(p) \sum_{i=1}^{\lambda_{p}} p^{i(2-n)+\frac{n-3}{2}}\right\} .
\end{aligned}
$$

Here $h=2^{v} g=2^{v} r^{2} t$ with $v$ a nonnegative integer, $r$ a positive integer, and $t$ a square-free integer. Furthermore: $B_{n-1}$ is a Bernoulli number, $\psi_{h}$ is the primitive Dirichlet character corresponding to the algebraic number field $\mathbb{Q}\left((-1)^{\frac{m}{2}} h^{\frac{1}{2}}\right)$, $L\left(s, \psi_{h}\right)$ is the $L$-function of $\psi_{h}, r=\prod_{p} p^{\lambda_{p}}$ is the prime decomposition of $r$, and $\tilde{b}(h)=q^{\nu}+\varphi_{1}(n) 2^{-\frac{1}{2}} \cdot \frac{q^{\nu+1}-2^{n-1}+1}{2^{n-2}-1}$ if $v$ is odd, $\tilde{b}(h)=q^{v}+\varphi_{1}(n) 2^{m-1} \cdot \frac{q^{\nu}-1}{q^{2}-1}+$ $\varphi_{1}(n) \varphi(n g) 2^{-m}+\varphi\left(\frac{g+2-n}{2}\right) 2^{2-n}$ if $\nu$ is even. 
In the expressions for $\tilde{b}(h), q$ stands for $2^{\frac{n-2}{2}}, \varphi_{1}(n)=(-1)^{\frac{n^{2}-1}{8}}$, and $\varphi$ is the primitive Dirichlet character modulo 4 , i.e., $\varphi(k)=0$ for even $k, \varphi(k)=1$ if $k \equiv$ $1 \bmod 4$, and $\varphi(k)=-1$ if $k \equiv 3 \bmod 4$.

For even $n=2 m>2$, the formulas for $A_{0}(I, h)$ are even more explicit. They are stated in [2], pp. 1074, 1075. Again we write $h=2^{v} g, v \geq 0$ and $g$ odd.

If $n \equiv 2 \bmod 4$, then

$$
A_{0}(I, h)=\frac{4}{E_{\frac{m-1}{2}}}\left\{\varphi(g) 2^{(v+1)(m-1)}+\varphi(m)\right\} \sum_{d \mid g} \varphi(d) d^{m-1},
$$

where $E_{\frac{m-1}{2}}$ is the $\frac{m-1}{2}$ th Euler number.

If $n \equiv 4 \bmod 8$, then

$$
A_{0}(I, h)=\frac{n}{\left(2^{m}-1\right)\left|B_{m}\right|} \cdot \sum_{d \mid g} d^{m-1} \cdot \begin{cases}1 & \text { if } v=0 \\ \sum_{i=0}^{v} 2^{i(m-1)}-2 \sum_{i=1}^{v-1} 2^{i(m-1)} & \text { if } v>0\end{cases}
$$

If $n \equiv 0 \bmod 8$, then

$$
A_{0}(I, h)=\frac{n}{\left(2^{m}-1\right)\left|B_{m}\right|} \cdot \begin{cases}\sum_{d \mid h} d^{m-1} & \text { if } v=0, \\ \sum_{d \mid h} d^{m-1}-2 \sum_{d \mid g} d^{m-1} & \text { if } v>0 .\end{cases}
$$

Again we look at the numbers $A_{0}\left(I, t k^{2}\right)$ where $k$ is a positive and $t$ an odd square-free integer. First we assume that $n$ is odd, and we use (5). From the definition of the character $\psi_{h}$ it follows that $\psi_{t k^{2}}=\psi_{t}$. If $k$ is odd, then $\tilde{b}\left(t k^{2}\right)=$ $1+\varphi_{1}(n) \varphi\left(n t k^{2}\right) 2^{-m}+\varphi\left(\frac{t k^{2}+2-n}{2}\right) 2^{2-n}$. Since $k^{2} \equiv 1 \bmod 8$, we get

$$
\tilde{b}\left(t k^{2}\right)=1+\varphi_{1}(n) \varphi(n t) 2^{-m}+\varphi\left(\frac{t+2-n}{2}\right) 2^{2-n}=\tilde{b}(t) .
$$

This results in the following expression for the quotient $\rho(I, t, k)=\frac{A_{0}\left(I, t k^{2}\right)}{A_{0}(I, t)}$ :

$$
\rho(I, t, k)=k^{n-2} \prod_{p \mid k}\left\{\sum_{i=0}^{\lambda_{p}} p^{i(2-n)}-\psi_{t}(p) \sum_{i=1}^{\lambda_{p}} p^{i(2-n)+\frac{n-3}{2}}\right\} .
$$

If $k=2^{v} g$ ( $g$ odd and $\left.v \geq 1\right)$ is even, then we do not have $\tilde{b}\left(t k^{2}\right)=\tilde{b}(t)$. In this case we have the following formula for $\rho(I, t, k)$ :

$$
\rho(I, t, k)=g^{n-2} \frac{\tilde{b}\left(t k^{2}\right)}{\tilde{b}(t)} \prod_{p \mid g}\left\{\sum_{i=0}^{\lambda_{p}} p^{i(2-n)}-\psi_{t}(p) \sum_{i=1}^{\lambda_{p}} p^{i(2-n)+\frac{n-3}{2}}\right\} .
$$

Next we suppose $n$ to be even, and we use (6), (7), and (8). 
Assume first that $k$ is odd and $n \equiv 2 \bmod 4$. Write $k=\prod_{p} p^{\lambda_{p}}$. We get

$$
\begin{aligned}
\sum_{d \mid t k^{2}} \varphi(d) d^{m-1}= & \prod_{p \mid t, p \nmid k}\left(1+\varphi(p) p^{m-1}\right) \prod_{p \mid \operatorname{gcd}(t, k)}\left(\sum_{i=0}^{2 \lambda_{p}+1} \varphi\left(p^{i}\right) p^{i(m-1)}\right) \\
& \times \prod_{p \mid k, p \nmid t}\left(\sum_{i=0}^{2 \lambda_{p}} \varphi\left(p^{i}\right) p^{i(m-1)}\right) \\
= & \prod_{p \mid t}\left(1+\varphi(p) p^{m-1}\right) \prod_{p \mid \operatorname{gcd}(t, k)}\left(\sum_{i=0}^{\lambda_{p}} p^{2 i(m-1)}\right) \\
& \times \prod_{p \mid k, p \nmid t}\left(\sum_{i=0}^{2 \lambda_{p}} \varphi\left(p^{i}\right) p^{i(m-1)}\right) .
\end{aligned}
$$

From (6) we see that

$$
\rho(I, t, k)=\prod_{p \mid \operatorname{gcd}(t, k)}\left(\frac{p^{(n-2)\left(\lambda_{p}+1\right)}-1}{p^{n-2}-1}\right) \prod_{p \mid k, p \nmid t, p \text { odd }}\left(\frac{\varphi(p) p^{(m-1)\left(2 \lambda_{p}+1\right)}-1}{\varphi(p) p^{m-1}-1}\right) .
$$

If $k=2^{v} \prod_{p} p^{\lambda}, v>0$, is even, this expression must be multiplied by the factor

$$
\frac{\varphi(t) 2^{(m-1)(2 v+1)}+\varphi(m)}{\varphi(t) 2^{m-1}+\varphi(m)} .
$$

We treat the cases $n \equiv 4 \bmod 8$ and $n \equiv 0 \bmod 8$ in the same way. We write $k=$ $2^{v} \prod_{p, p \text { odd }} p^{\lambda p}$.

For $n \equiv 4 \bmod 8$, we get

$$
\begin{aligned}
\rho(I, t, k)= & \prod_{p \mid \operatorname{gcd}(t, k)}\left(\frac{p^{(n-2)\left(\lambda_{p}+1\right)}-1}{p^{n-2}-1}\right) \\
& \times \prod_{p \mid k, p \nmid t, p \text { odd }}\left(\frac{p^{(m-1)\left(2 \lambda_{p}+1\right)}-1}{p^{m-1}-1}\right) \cdot \begin{cases}1 & \text { if } \nu=0, \\
\epsilon_{1}(v) & \text { if } \nu>0,\end{cases}
\end{aligned}
$$

where $\epsilon_{1}(v)=\frac{2^{v(n-2)}\left(2^{m-1}-2\right)+2^{m}-1}{2^{m-1}-1}$.

And for $n \equiv 0 \bmod 8$, we get

$$
\begin{aligned}
\rho(I, t, k)= & \prod_{p \mid \operatorname{gcd}(t, k)}\left(\frac{p^{(n-2)\left(\lambda_{p}+1\right)}-1}{p^{n-2}-1}\right) \\
& \times \prod_{p \mid k, p \nmid t, p \text { odd }}\left(\frac{p^{(m-1)\left(2 \lambda_{p}+1\right)}-1}{p^{m-1}-1}\right) \cdot \begin{cases}1 & \text { if } v=0, \\
\epsilon_{2}(v) & \text { if } v>0,\end{cases}
\end{aligned}
$$

where $\epsilon_{2}(v)=\frac{2^{(m-1)(2 v+1)}-2^{m}+1}{2^{m-1}-1}$. 
The following step is to compute $\sum_{j=0}^{\infty} \frac{\rho(I, t, k)}{p^{j s}}$. Again we assume first that $n$ is odd. Using (9), we find that, for any odd prime $p$,

$$
\sum_{j=0}^{\infty} \frac{\rho\left(I, t, p^{j}\right)}{p^{j s}}=\frac{1-\frac{\delta(p)}{p^{s}}}{\left(1-\frac{1}{p^{s}}\right)\left(1-\frac{1}{p^{s+2-n}}\right)}
$$

where $\delta(p)=\psi_{t}(p) p^{\frac{n-3}{2}}$. For the prime $p=2$, we get a more complicated expression,

$$
\sum_{j=0}^{\infty} \frac{\rho\left(I, t, 2^{j}\right)}{2^{j s}}=T_{n}(s) \frac{1}{\left(1-\frac{1}{p^{s}}\right)\left(1-\frac{1}{p^{s+2-n}}\right)},
$$

where $T_{n}(s)=1-\frac{1-\varphi_{1}(n) 2^{m-1}+\varphi_{1}(n) \varphi(n t) 2^{m-1}+\varphi\left(\frac{t+2-n}{2}\right)}{1+\varphi_{1}(n) \varphi(n t) 2^{-m}+\varphi\left(\frac{t+2-n}{2}\right) 2^{2-n}} \cdot \frac{1}{2^{s}}$. From these expressions we get that for odd $n$, we have

$$
\sum_{k=1}^{\infty} \frac{\rho(I, t, k)}{k^{s}}=T_{n}(s) \prod_{p \text { odd }}\left(1-\frac{\delta(p)}{p^{s}}\right) \zeta(s) \zeta(s+2-n)
$$

If $n$ is even, we can proceed in the same way, and using the expressions (11), (12), and (13) in the various cases, we get similar expressions. We summarize our results in the following theorem.

\section{Theorem 3 Let $n \geq 4$.}

(i) If $n$ is odd, then

$$
\sum_{k=1}^{\infty} \frac{\rho(I, t, k)}{k^{s}}=T_{n}(s) \prod_{p \text { odd }}\left(1-\frac{\delta(p)}{p^{s}}\right) \zeta(s) \zeta(s+2-n) .
$$

(ii) If $n$ is even, then

$$
\sum_{k=1}^{\infty} \frac{\rho(I, t, k)}{k^{s}}=T_{n}(s) \prod_{p \nmid t, p \text { odd }}\left(1+\frac{\varphi(p) p^{m-1}}{p^{s}}\right) \zeta(s) \zeta(s+2-n),
$$

where
(a) $T_{n}(s)=1-\frac{1}{2^{s}} \cdot \frac{\varphi(t) 2^{m-1}+\varphi(m) 2^{2 m-2}}{\varphi(t) 2^{m-1}+\varphi(m)}$ if $n \equiv 2 \bmod 4$,
(b) $T_{n}(s)=1-\frac{2^{m-1}}{2^{s}}-\frac{2^{2 m-1}}{2^{2 s}}$ if $n \equiv 4 \bmod 8$,
(c) $T_{n}(s)=1+\frac{2^{m-1}-2}{2^{s}}+\frac{2^{2 m-1}}{2^{2 s}}$ if $n \equiv 0 \bmod 8$.

Now we look in particular at the case where $2 \leq n \leq 8$ and $t=1$. For $2 \leq n \leq 8$, there is only one class in the genus of the quadratic form $Q(X)=X^{T} X$ (see, for instance, [2]). This means that $A_{0}\left(I, k^{2}\right)=r_{n}\left(k^{2}\right)$, the number of representations of $k^{2}$ as a sum of $n$ squares. Besides, $A_{0}(I, 1)=2 n$, so we get $\rho(I, 1, k)=\frac{1}{2 n} r_{n}\left(k^{2}\right)$. So far in this section we assumed that $n \geq 4$. However, there is a rather simple expression for 
$r_{2}(k)$, which can, for instance, be found in [2], too. If we write $k=2^{v} g=2^{v} \prod_{p} p^{\lambda_{p}}$, then

$$
r_{2}(k)=2(1+\varphi(g)) \sum_{d \mid g} \varphi(d) .
$$

The expression for $r_{3}(k)$ is more complicated, but for a square $k^{2}$, we have

$$
r_{3}\left(k^{2}\right)=6 \prod_{p \text { odd }}\left(\frac{p^{\lambda_{p}+1}-1}{p-1}-\varphi(p) \frac{p^{\lambda_{p}}-1}{p-1}\right) .
$$

This formula can also be found in [9].

Using the results of Theorem 3 and the two formulas for $r_{2}$ and $r_{3}$, we get as a special case the following theorem.

Theorem 4 For $2 \leq n \leq 8$, we have

$$
\sum_{k=1}^{\infty} \frac{r_{n}\left(k^{2}\right)}{k^{s}}=2 n T_{n}(s) \prod_{p \neq 2}\left\{1+\frac{(-1)^{n}\left(\left(\frac{-1}{p}\right) p\right)^{\left\lfloor\frac{n}{2}\right\rfloor}}{p^{s+1}}\right\} \zeta(s) \zeta(s+2-n),
$$

where $T_{n}(s)$ is defined by

$$
\begin{cases}1-\frac{1}{2^{s}} & \text { if } n=2, \\ 1-\frac{2}{2^{s}} & \text { if } n=3, \\ 1-\frac{2}{2^{s}}-\frac{8}{2^{2 s}} & \text { if } n=4, \\ 1 & \text { if } n=5, \\ 1+\frac{4}{2^{s}} & \text { if } n=6, \\ 1+\frac{8}{2^{s}} & \text { if } n=7 \\ 1+\frac{6}{2^{s}}+\frac{128}{2^{2 s}} & \text { if } n=8\end{cases}
$$

\section{Indefinite quadratic forms}

In this section we consider the case where $S$ is nonsingular but indefinite, i.e., the quadratic form $X^{T} S X$ takes both positive and negative values. The genus of such a quadratic form still consists of a finite number of classes. The problem compared to Sect. 3 is that in this case there is no analog to the factor $\alpha_{\infty}(S, t)$ : the real solution space of $X^{T} S X=t$ does not have a finite volume. In [5] and [6] Siegel introduced a measure $\mu(S, t)$ on the solution space of $X^{T} S X=t$ for this case. Let $\sigma$ be the signature of $S$, i.e., the number of positive minus the number of negative eigenvalues of $S$. The following proposition is a special case of [6], Theorem 1.

Proposition 2 If $n>4$ and $0<\sigma<n$, we have $\mu(S, t)=\prod_{p} \alpha_{p}(S, t)$. 
Next, analogously to the definite case, we put

$$
\rho(S, t, k)=\frac{\mu\left(S, t k^{2}\right)}{\mu(S, t)}=\prod_{p \mid k} \frac{\alpha_{p}\left(S, t k^{2}\right)}{\alpha_{p}(S, t)} .
$$

Now we can proceed in the same way as in Sect. 3. Since the factor $k^{n-2}$ in the expression for $\rho(S, t, k)$ is missing, in this case we will get slightly different expressions. For the bad primes $p$ which divide $2|S|$, we write $G(p, 2|S|)=$ $\left(1-\frac{1}{p^{s}}\right)\left(1-\frac{1}{p^{s+n-2}}\right) \sum_{j=0}^{\infty} \frac{\rho\left(S, t, p^{j}\right)}{p^{j s}}$. As a result, we finally get the following theorem.

Theorem 5 If $n>4$ and $0<\sigma<n$, then we have

$$
\begin{aligned}
\sum_{k=1}^{\infty} \frac{\rho(S, t, k)}{k^{s}}= & \prod_{p|2| S \mid} G(p, 2|S|) \\
& \times \prod_{p \nmid 2|S| t}\left(1+(-1)^{n} \frac{\left(\frac{(-1)^{\left\lfloor\frac{n}{2}\right\rfloor}|S| t^{n}}{p}\right) p^{-\left\lfloor\frac{n-1}{2}\right\rfloor}}{p^{s}}\right) \zeta(s) \zeta(s+n-2) .
\end{aligned}
$$

Open Access This article is distributed under the terms of the Creative Commons Attribution Noncommercial License which permits any noncommercial use, distribution, and reproduction in any medium, provided the original author(s) and source are credited.

\section{References}

1. Hardy, G.H.: Ramanujan. Cambridge University Press, Cambridge (1949)

2. Shimura, G.: The representation of integers as sums of squares. Am. J. Math. 124, 1059-1081 (2002)

3. Shimura, G.: On modular forms of half integral weight. Ann. Math. 97, 440-481 (1973)

4. Siegel, C.L.: Ueber die analytische Theorie der quadratischen Formen. Ann. Math. 36, 527-606 (1935)

5. Siegel, C.L.: Ueber die analytische Theorie der quadratischen Formen II. Ann. Math. 37, 230-263 (1936)

6. Siegel, C.L.: On the theory of indefinite quadratic forms. Ann. Math. 45, 577-622 (1944)

7. Andrianov, A.N., Zhuravlev, V.G.: Modular Forms and Hecke Operators. Translations of Mathematical Monographs, vol. 145. AMS, Providence (1991)

8. Zhuravlev, V.G.: Multiplicative arithmetic of theta-series of odd quadratic forms. Izv. Math. 59(3), 517-578 (1995)

9. Cooper, S.: Sums of five, seven and nine squares. Ramanujan J. 6, 469-490 (2002) 